\title{
Concepto de calidad de vida en mujeres con cáncer
}

\author{
The concept of quality of life in women suffering from cancer
}

\author{
Ricardo Sánchez-Pedraza ${ }^{1,2}$, Mónica P. Ballesteros ${ }^{1}$ y Jorge D. Anzola ${ }^{1}$ \\ 1 Universidad Nacional de Colombia, Instituto Nacional de Cancerología, Bogotá, DC. \\ rsanchezpe@unal.edu.co \\ 2 Instituto Nacional de Cancerología, Bogotá, DC. \\ Recibido 23 Febrero 2010/Enviado para Modificación 12 Noviembre 2010/Aceptado 29 Noviembre 2010
}

RESUMEN

Objetivo Analizar el concepto de calidad de vida en mujeres con cáncer y evaluar la estructura del concepto según grupos de edad.

Material y métodos Se efectuó esta pregunta abierta a 114 mujeres con cáncer de seno: ¿En su opinión, qué es calidad de vida? Se realizó análisis lexicográfico de las respuestas mediante métodos factoriales para variables categóricas.

Resultados Las formas gráficas y los segmentos repetidos más frecuentes se relacionaron con conceptos asociados a bienestar y salud. El análisis de formas características corrobora la importancia de conceptos relacionados con salud, bienestar y tranquilidad. Según los grupos de edad hay conceptos predominantes: En el de mujeres más jóvenes destaca la preocupación por el derecho de tener buen tratamiento. En el de 41-50 años el poder manejar su enfermedad y ver respuesta al tratamiento. En el de 51-60 años es importante la capacidad funcional y de disfrute, a pesar de padecer enfermedad. En el de mayores de 60 años resalta el bienestar espiritual. Se detectaron tres dimensiones: Salud como capacidad de disfrutar funciones nutricias y afectivas, capacidad de autonomía o de sana dependencia, y armonía familiar y social. Los grupos de edad se representan de manera diferencial en cada una de estas tres dimensiones.

Conclusiones La espiritualidad y la disponibilidad de sistemas de salud que garanticen el tratamiento surgen como dominios importantes en pacientes con cáncer. La calidad de vida es un concepto que se modifica dependiendo de la edad de los pacientes, lo cual apoya la concepción subjetiva del constructo.

Palabras Clave: Calidad de vida, evaluación en salud, formación de concepto, salud, neoplasias, grupos por edad (fuente: DeCS, BIREME).

\section{ABSTRACT}

Objective Analyzing a group of females suffering from cancer's concept of the quality of life and evaluating how they perceived such concept, depending on patients' age-group.

Methods The following open-ended question was put to a sample of 114 women suffering from breast cancer, "In your opinion, what is quality of life?" Their replies were analyzed using factorial methods for categorical variables. 
Results The most used graphical forms and repeated segments were associated with notions linked to well-being and health; analyzing characteristic forms supported these concepts' importance. Some concepts appeared to be more relevant according to patient age-group. The young female group emphasized the right and opportunity to have adequate treatment whilst overcoming disease and response to its treatment were relevant to the 41-50 year-old group. Functioning and enjoyment were significant for the 51-60 year-old group whilst patients aged over 60 emphasized spiritual well-being. Binary correspondence analysis identified three domains: health (not just being the absence of disease or infirmity), autonomy or adequate dependence, and family/social well-being. Age-groups had differential representation regarding each domain.

Conclusions Besides traditional domains, spirituality and a particular system guaranteeing access to quality health-care emerged as being important domains in female cancer patients' perception of the quality of life. Quality of life is a multidimensional and dynamic concept that changes according to age; this suggested that quality of life is supported by subjective conceptualization; the use of scales for measuring quality of life could thus be questionable because of their subjectivity and dynamic nature.

Key Words: Quality of life, outcome assessment (health care), concept formation, health, neoplasm, age group (source: $M e S H, N L M$ ).

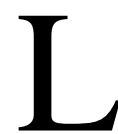

a calidad de vida es un concepto relativamente reciente dentro del ámbito del cuidado de la salud pero que ha venido ganando peso en la investigación clínica, a tal punto que en la actualidad es el segundo desenlace más importante después de la supervivencia en pacientes con cáncer (1). Esto se vincula con el hecho de que la concepción de salud y de cuidado de la salud son fenómenos cambiantes, lo cual se relaciona con dos factores: la aceptación de la importancia de las consecuencias sociales de la enfermedad y el reconocimiento de que las intervenciones en salud buscan prolongar la vida y la calidad de la misma (2). La creciente importancia que ha venido ocupando este desenlace se ha relacionado con el aumento en la expectativa de vida, lo que supone una mayor cantidad de pacientes padeciendo enfermedades crónicas y terminales (3), y con una mayor participación de los pacientes en la toma de decisiones sobre su enfermedad y tratamiento. Adicionalmente, la calidad de vida se constituye en un indicador de la calidad del cuidado que un paciente recibe en salud $(4,5)$. Dentro de los diferentes tipos de neoplasias el cáncer de mama es el que tiene mayor cantidad de ensayos clínicos en los que se ha medido la calidad de vida con instrumentos estandarizados (6). A pesar de la incorporación cada vez más frecuente de este constructo dentro de los desenlaces médicos, no existe un acuerdo unánime en cuanto a su conceptualización y definición (7). Este nivel de 
ambigüedad ha hecho que algunos autores se refieran al constructo como una "sombrilla" que cobija conceptos disímiles tales como funcionamiento, síntomas, comportamiento, percepciones, felicidad y condiciones de vida (8).

Las definiciones que se han planteado no necesariamente son comparables: Para algunos autores la calidad de vida representa la capacidad del paciente para resolver los problemas que se relacionan con el hecho de estar gravemente enfermo (9); Young la define como el grado de satisfacción percibida con las circunstancias vitales actuales (10); la OMS plantea un constructo multidimensional (salud física, estado psicológico, nivel de independencia, relaciones sociales, medio ambiente y preocupaciones espirituales) en el que se tienen en cuenta las percepciones individuales en el contexto cultural y el sistema de valores (11). Otras propuestas de multidimensionalidad plantean dominios tales como capacidad para vivir una vida normal, felicidad-satisfacción, logro de metas personales, capacidad para llevar una vida socialmente útil y capacidades físicas y mentales $(4,12,13)$. La falta de claridad en el concepto se refleja, obviamente, en las estrategias empleadas para su medición, campo en el cual tampoco hay consenso. Algunos estudios han reportado que no existe una correlación entre el estado de salud y la calidad de vida: en este sentido, es posible que pacientes presentando compromiso importante de su estado de salud refieran una adecuada calidad de vida, o viceversa (buenas condiciones de salud - mala calidad de vida) (14,15): este hallazgo se ha denominado "paradoja de discapacidad" y se ha reportado en pacientes con cáncer al encontrar que en escalas como el cuestionario de calidad de vida de la OMS (WHOQOL) éstos tienen puntajes mayores que muchos otros tipos de pacientes, incluso aquellos que asistían a una clínica de planificación familiar (16). Se ha reportado también que existen diferencias importantes entre la calificación de calidad de vida dada por el paciente, por el médico y por sus familiares (17). Adicionalmente, existen reportes de que los conceptos de calidad de vida cambian de acuerdo con la edad de los pacientes (18). Teniendo en cuenta lo anterior, se ha propuesto el término "Calidad de vida relacionada con la salud" como una manera de describir aspectos de la experiencia subjetiva de una persona, vinculados directa e indirectamente con la salud, la enfermedad, el tratamiento, la discapacidad y el deterioro (2).

Esta subjetividad abarca un componente cognoscitivo, relacionado con la apreciación del nivel de funcionamiento, y uno emocional relacionado con el grado de satisfacción experimentado por el paciente (19). Para algunos autores este último componente de satisfacción es más importante dentro 
del constructo (20), aunque para otros los aspectos comunitarios son variables centrales en la definición del concepto (21). La indefinición del concepto ha hecho que se utilicen indistintamente los términos "estado de salud", "calidad de vida", "calidad de vida relacionada con la salud" y "estado funcional" (22). Considerando tal nivel de imprecisión, algunos autores plantean que antes de determinar si un instrumento mide lo que queremos medir (validez), debe precisarse qué es lo que queremos medir (19). Para algunos autores el uso de métodos científicos para medir calidad de vida está todavía en una fase primitiva y el hecho de que no exista un patrón de oro aceptado impone cautela a la hora de aplicar e interpretar los resultados de estos instrumentos de medición (4). Los intentos para generar instrumentos que puedan ser usados en diferentes escenarios clínicos, e incluso en diferentes culturas, pasan por la necesidad de reconocer que el constructo no puede ser explicado por un modelo unidimensional $(23,24)$. Los instrumentos desarrollados para medir calidad de vida se enmarcan dentro de los siguientes aspectos (25):

- La medición de la calidad de vida busca que el tratamiento se enfoque más en el paciente que en la enfermedad.

- La puesta en práctica y los resultados de la medición pueden ser útiles para mejorar la relación médico-paciente y la calidad en el cuidado de la salud.

- Las medidas desarrolladas para evaluar calidad de vida en escenarios de investigación clínica no siempre resultan útiles en la práctica clínica convencional.

Al aceptarse que la calidad de vida es un constructo centrado en el paciente, surge un reto para su medición ya que se reconoce su carácter único en cada sujeto, lo cual impone grandes dificultades a la hora de generar instrumentos de medición estandarizados. Se ha venido cuestionando si tales instrumentos estandarizados realmente se centran en la individualidad del paciente, concepto medular en la definición de calidad de vida, más si se tiene en cuenta que este constructo yace en la experiencia vital, individual de la persona. Se plantea que tales instrumentos no necesariamente tienen en cuenta lo que es importante para los pacientes a la hora de medir este constructo; por ejemplo, en un estudio se reportó que el aspecto que más influía sobre la calidad de vida de un grupo de pacientes con cáncer era la dificultad de encontrar parqueadero cuando asistían a sus consultas (26). Los indicadores objetivos generados por escalas de medición describen algunas condiciones vitales que tienen repercusión en la biografía del paciente; sin embargo, no evalúan directamente experiencias subjetivas (27). En este 
sentido resulta cuestionable que los instrumentos estandarizados evalúen de manera valida y confiable el constructo: tales instrumentos estarían midiendo lo que los investigadores, profesionales o la sociedad creen que es la calidad de vida para las personas que están enfermas, lo cual no necesariamente corresponde a los aspectos más prioritarios para el paciente (28). Esto lo han demostrado estudios cualitativos en los que se encuentra que las dimensiones que incorporan los instrumentos de medición no siempre coinciden con los que perciben los pacientes como importantes (28). Además, se han cuestionado los métodos de ponderación que los instrumentos utilizan en los diferentes dominios, ya que diferentes grupos de pacientes pueden dar diferente peso a las distintas dimensiones del constructo (28). Adicionalmente se ha reportado que las respuestas que da un paciente si se le pregunta abiertamente sobre algún aspecto de su enfermedad difieren de aquellas que ofrece ante una escala estructurada que explora los mismos aspectos (29). Teniendo en cuenta los anteriores cuestionamientos relacionados con los instrumentos estandarizados se ha propuesto que se apliquen medidas individualizadas como el esquema para evaluación individualizada de calidad de vida (SEIQOL) (30), el índice generado por el paciente (PGI) (16), el perfil de repercusión de enfermedad (DRP)(31), o el perfil subjetivo de calidad de vida (SQLP)(32). Sin embargo se ha cuestionado la dificultad para la interpretación y análisis de los datos producidos por estas mediciones subjetivas (28).

Teniendo en cuenta que el cáncer de mama en Colombia es un problema importante de salud (actualmente este tipo de cáncer es la segunda causa de muerte entre mujeres), que dicha mortalidad presenta tendencias crecientes en el país (33), y que es un problema de salud que compromete de manera significativa la calidad de vida de las pacientes, en el presente estudio se planteó como objetivo analizar el concepto de calidad de vida en un grupo de mujeres con esta patología, a partir de un análisis lexicográfico, y evaluar la estructura del concepto dependiendo del grupo de edad de las pacientes. Este objetivo se fundamenta en los siguientes aspectos:

- Es importante conocer la subjetividad del paciente en relación con este concepto, teniendo en cuenta los cuestionamientos que se han hecho a los instrumentos estandarizados para medir el constructo.

- Se han reportado estudios en pacientes con cáncer de mama que sugieren variabilidad del concepto de calidad de vida, en relación con variables como evolución de la enfermedad y edad de las pacientes $(34,35)$. 


\section{MATERIAL Y MÉTODOS}

Se efectuó una pregunta abierta a 210 mujeres con diagnóstico de cáncer de mama que asistían a diagnóstico o tratamiento en servicios ambulatorios en el INC durante los años 2006 a 2009. 114 de las pacientes respondieron a la pregunta formulada (¿Qué es para usted calidad de vida?). La respuesta fue transcrita en un formato de texto plano y posteriormente exportada al programa SPAD 5® para realizar análisis lexicográfico. Para este análisis se considera que el conjunto de palabras (aquí denominadas "formas gráficas") conforman un vocabulario. Para efectuar la reducción y delimitación del vocabulario se tomaron los siguientes criterios (36):

1. Eliminar palabras "herramientas": artículos, preposiciones, conjunciones y pronombres.

2. Tomar un umbral de frecuencia de tres para eliminar palabras de menos de esa longitud.

Para efectuar el análisis se tomó como variable de agrupación la edad categorizada así: De 30 a 40 años (8 pacientes), de 41 a 50 (31 pacientes), de 51 a 60 (34 pacientes) y mayores de 60 (41 pacientes).

Para cada categoría se efectuó una selección de formas características (Son respuestas reales dadas por los pacientes, que son representativas de una categoría de individuos). Para esto se escogió el criterio de valores-test usando elementos característicos (respuestas que contienen la mayoría de palabras que son más características del grupo) (37). El presente estudio fue aprobado por el comité de Ética en Investigaciones del Instituto Nacional de Cancerología.

\section{RESULTADOS}

El análisis lexicográfico de la pregunta: "Para usted, ¿Qué es calidad de vida?" arrojó un total de 114 respuestas con 2088 formas gráficas (tamaño del vocabulario) y 584 formas diferentes (formas gráficas no repetidas). La riqueza del vocabulario (tamaño del vocabulario/formas gráficas no repetidas) se cuantificó en un $28 \%$.

Al aplicar los criterios de reducción mencionados se conservó un total de 171 formas distintas. Las formas gráficas más utilizadas se presentan en la Tabla 1. Los segmentos repetidos más frecuentes se relacionaron con 
conceptos asociados a bienestar y salud (Tabla 2). Según las categorías de edad, la cantidad de formas se repartió de manera proporcional al tamaño de cada uno de los grupos (Tabla 3). Los resultados de la selección de formas características para cada uno de los grupos de edad definidos se presentan en la Tabla 4. Como puede observarse, el común denominador en los diferentes grupos se relaciona con la salud, el bienestar y la tranquilidad.

Tabla 1. Formas gráficas más utilizadas

\begin{tabular}{lcc}
\hline \multicolumn{1}{c}{ Palabra } & Frecuencia & Longitud \\
\hline Bien & 53 & 4 \\
Tener & 48 & 5 \\
Salud & 37 & 5 \\
Estar & 35 & 5 \\
Vida & 34 & 4 \\
Buena & 20 & 5 \\
Sin & 20 & 3 \\
Vivir & 17 & 5 \\
Dios & 14 & 4 \\
Hacer & 13 & 5 \\
Poder & 13 & 5 \\
Tratamiento & 13 & 11 \\
Todo & 12 & 4 \\
Familia & 12 & 7 \\
Disfrutar & 12 & 9 \\
Sentirme & 10 & 8 \\
Sentirse & 10 & 8 \\
\hline
\end{tabular}

Tabla 2. Segmentos repetidos por orden de frecuencia

\begin{tabular}{|cc|}
\hline Frecuencia & Segmento \\
\hline 20 & Estar bien \\
\hline 16 & Sentir bien \\
10 & Puena salud \\
\hline 5 & Tener salud \\
5 & Bien sentir \\
\hline 5 & Vivir bien \\
\hline 4 & Buen comer \\
4 & Vivir normal \\
3 & Poder disfrutar \\
3 & Estar bien sentir \\
\hline 3 & Tener buena \\
3 & Salud bienestar \\
2 & Vivir salud \\
2 & Poder comer \\
2 & Tener dolor \\
2 & Vivir tranquila \\
\hline 2 & Estar tranquila \\
\hline 2 & Tratamiento médico \\
\hline 2 & Tener buena salud \\
\hline 2 & Tener vida \\
2 & Tener tranquilidad \\
\hline 2 & Estar bien sentirse bien \\
\hline
\end{tabular}


En el grupo de menores de 40 años se destaca la preocupación por el derecho y la oportunidad de tener un buen tratamiento. En el grupo de 41 a 50 años se resalta la posibilidad de poder manejar la enfermedad y ver la respuesta al tratamiento. En el grupo de 51 a 60 años tiene importancia la capacidad funcional y de disfrute, a pesar de padecer enfermedad. En el grupo de mayores de 60 años se resalta la importancia del bienestar espiritual.

Tabla 3. Distribución del total de formas según grupos de edad

\begin{tabular}{lcccc}
\hline Edad & $\mathrm{N}$ & $\begin{array}{c}\text { Total de } \\
\text { formas }\end{array}$ & \% del total & $\begin{array}{c}\text { \# de formas } \\
\text { diferentes }\end{array}$ \\
\hline $30-40$ & 8 & 210 & 9,57 & 40 \\
$41-50$ & 31 & 712 & 32,44 & 62 \\
$51-60$ & 34 & 511 & 23,28 & 62 \\
$>60$ & 41 & 762 & 34,72 & 68 \\
Total & & 2195 & 100 & \\
\hline
\end{tabular}

Tabla 4. Formas características según grupo de edad

\begin{tabular}{|c|c|}
\hline Grupo & Forma característica \\
\hline \multirow{4}{*}{30 a 40} & Tener vida sana y tranquila \\
\hline & Sentirme bien en todos los aspectos, sentir que lo que me rodea me da sentido devida. \\
\hline & $\begin{array}{l}\text { Tener los mejores tratamientos médicos, psicológicos y emocionales que puedan haber } \\
\text { para tener un ritmo de vida normal }\end{array}$ \\
\hline & $\begin{array}{l}\text { Tener derecho a la salud, especialmente a tener un buen tratamiento, tener una buena } \\
\text { alimentación, vivir en armonia con las personas que nos rodean y estar en paz conDios }\end{array}$ \\
\hline \multirow{4}{*}{41 a 50} & $\begin{array}{l}\text { Aprender a manejar la enfermedad de la mejor manera teniendomínimo dolor y los } \\
\text { mejores resultados }\end{array}$ \\
\hline & $\begin{array}{l}\text { No tener ninguna clase de enfermedades en ninguna parte del cuerpo ni de la mente } \\
\text { No vivir enferma: ver que el tratamiento si sirvió }\end{array}$ \\
\hline & $\begin{array}{l}\text { Continuar mi vida lo mejor posible o sea las actividades que venia realizando } \\
\text { continuarlas igual aunque soy consciente del cuidado que debo tener. Las alteraciones } \\
\text { por el tratamiento que sean lo menos posible }\end{array}$ \\
\hline & Vivir tranquila, sin muchos problemas o dificultades, estar en paz \\
\hline \multirow{6}{*}{51 a 60} & $\begin{array}{l}\text { Poder disfrutar de todo lo que la vida nos ofrece. poder comer, dormir, descansar, gozar, } \\
\text { a la medida de nuestros medios económicos. Disfrutar de su familia. Ya con la } \\
\text { enfermedad que llevamos a cuestas tratar de vivir y fuera de presión }\end{array}$ \\
\hline & $\begin{array}{l}\text { Estar bien, tener la capacidad de hacer las mismas cosas que hacia antes de saber de } \\
\text { mi enfermedad sin depender de nadie. Es tener buena salud a pesar de mi enfermedad } \\
\text { y saber que todo el tratamiento no ha afectado mucho mi vida cotidiana, ya que los } \\
\text { efectos secundarios no han causado mucho daño en mi vida }\end{array}$ \\
\hline & Tener la salud, porque con la salud puede ser uno pobre \\
\hline & No sentir dolor, poder comer, poder dormir, poder hacer mis cosas cotidianas \\
\hline & $\begin{array}{l}\text { Estar en perfecto estado de salud porque de ahidependen todas nuestras actividades } \\
\text { que nos llevan a ser felices }\end{array}$ \\
\hline & Estar bien y poder valerme por mi misma \\
\hline \multirow[b]{2}{*}{$>60$} & $\begin{array}{l}\text { Tener una vida tranquila en paz. Estar unida a Dios y guiada por él para sentirse bien } \\
\text { espiritual y fisicamente }\end{array}$ \\
\hline & $\begin{array}{l}\text { Tener muy buena salud, tener unión con la familia, servir a los demás, amar a la gente } \\
\text { Estar física, psicológica y espiritualmente bien } \\
\text { El amor, apoyo moral, el saber que los tiene al lado y que como sea podré salir adelante }\end{array}$ \\
\hline
\end{tabular}


Adicionalmente se efectuó un análisis de correspondencias binarias a partir de una tabla léxica de contingencia de 219 filas (171 formas simples, 48 segmentos repetidos) y cuatro columnas (categorías de edad). La reducción de dimensionalidad se efectuó con base en los cambios de inercia en un histograma de valores propios, arrojando una solución de tres factores. La selección de formas léxicas integrantes de cada eje se realizó con base en los valores de contribución al eje y los cosenos cuadrados. Los resultados se muestran en la Tabla 5 y en las Figuras 1 y 2 . El eje 1 representa la dicotomía salud-enfermedad: En el polo positivo del eje se representa una concepción básica en la que se relacionan salud con funciones nutricias y afectivas. En el polo positivo del eje se proyecta el grupo de mujeres de 51 a 60 años. En el polo negativo se representa la concepción negativa de calidad de vida, donde la enfermedad y el dolor quisieran evitarse, reducirse o mejorarse. En este polo se proyecta el grupo de mujeres de 41 a 50 años. El eje 2 incorpora un concepto de autonomía-dependencia: En el polo positivo del eje se agrupan ideas relacionadas con la capacidad de vivir una vida normal dentro de la cotidianidad. En este polo se proyecta el grupo de mujeres de 51 a 60 años. El polo negativo se relaciona con necesidades de atención, ayuda y comprensión para poder hacer las cosas. Este concepto de dependencia se relaciona con la idea de vínculos en el hogar. En este polo se proyectan las mujeres mayores de 60 años. El eje tres puede relacionarse con los conceptos de apoyo familiar y social como indicadores de calidad de vida. En el polo positivo del eje se localizan ideas de sentir y tener calidad, tranquilidad y bienestar al estar rodeado de personas. Aquí se proyecta el grupo de mujeres de 30 a 40 años. En el polo negativo se ubican conceptos de gusto relacionados con propósitos más activos (trabajar, decir, seguir). En este polo se proyecta el grupo de mujeres de 51 a 60 años.

Tabla 5. Resultados del análisis de correspondencias binarias

\begin{tabular}{|c|c|c|c|c|c|}
\hline \multicolumn{2}{|c|}{ Eje 1} & \multicolumn{2}{|c|}{ Eje 2} & \multicolumn{2}{|c|}{ Eje 3} \\
\hline $\begin{array}{c}51 \text { a } 60 \\
(+)\end{array}$ & $\begin{array}{c}41 \text { a } 50 \\
(-)\end{array}$ & $\begin{array}{c}51 \text { a } 60 \\
(+)\end{array}$ & $\begin{array}{c}>60 \\
(-)\end{array}$ & $\begin{array}{c}30 \text { a } 40 \\
(+)\end{array}$ & $\begin{array}{c}51 \text { a } 60 \\
(-)\end{array}$ \\
\hline salud & mejor & llevar & atención & rodea & mucho \\
\hline alimentación & enfermedad & poder & ayuda & mayor & decir \\
\hline amar & dificultades & ser & casa & bueno & gusta \\
\hline $\begin{array}{l}\text { poder } \\
\text { alimentarse }\end{array}$ & $\begin{array}{l}\text { posible } \\
\text { menor } \\
\text { activa } \\
\text { ninguna } \\
\text { no tener } \\
\text { tener dolor } \\
\text { poder hacer }\end{array}$ & $\begin{array}{l}\text { vivir } \\
\text { ser tener } \\
\text { tener bueno } \\
\text { vida cotidiana } \\
\text { vivir normal }\end{array}$ & $\begin{array}{l}\text { comprensión } \\
\text { espiritualmente } \\
\text { físicamente } \\
\text { futuro } \\
\text { unión } \\
\text { bien casa } \\
\text { hacer todo }\end{array}$ & $\begin{array}{l}\text { calidad } \\
\text { tener } \\
\text { personas } \\
\text { sin dificultades } \\
\text { sentir } \\
\text { buena salud }\end{array}$ & $\begin{array}{l}\text { trabajar } \\
\text { seguir }\end{array}$ \\
\hline
\end{tabular}




\section{DISCUSIÓN}

En el grupo de pacientes que participó en este estudio los conceptos de bienestar y de gozar de buena salud son los más frecuentemente relacionados con calidad de vida. Según los resultados, la salud se incorpora como un aspecto importante dentro de este concepto, lo cual estaría a favor del constructo denominado "calidad de vida relacionada con la salud", el cual propone medir aspectos de la experiencia subjetiva de un individuo relacionados con la salud, la enfermedad, la discapacidad y el deterioro (2).

Figura 1. Análisis de correspondencias binarias, factores 1 y 2

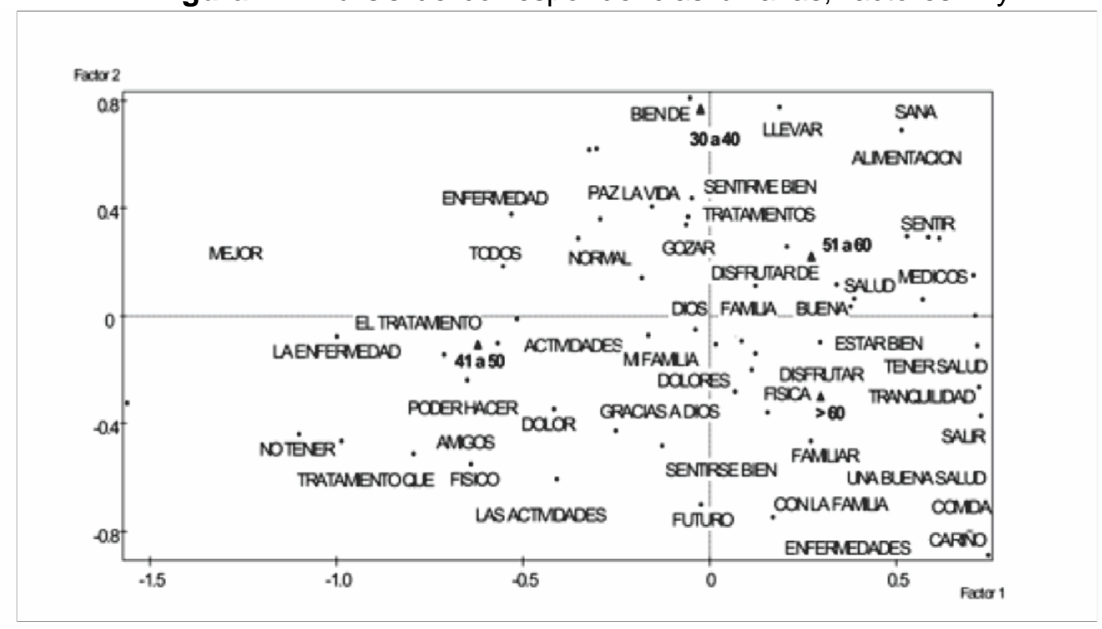

Figura 2. Análisis de correspondencias binarias, factores 1 y 3

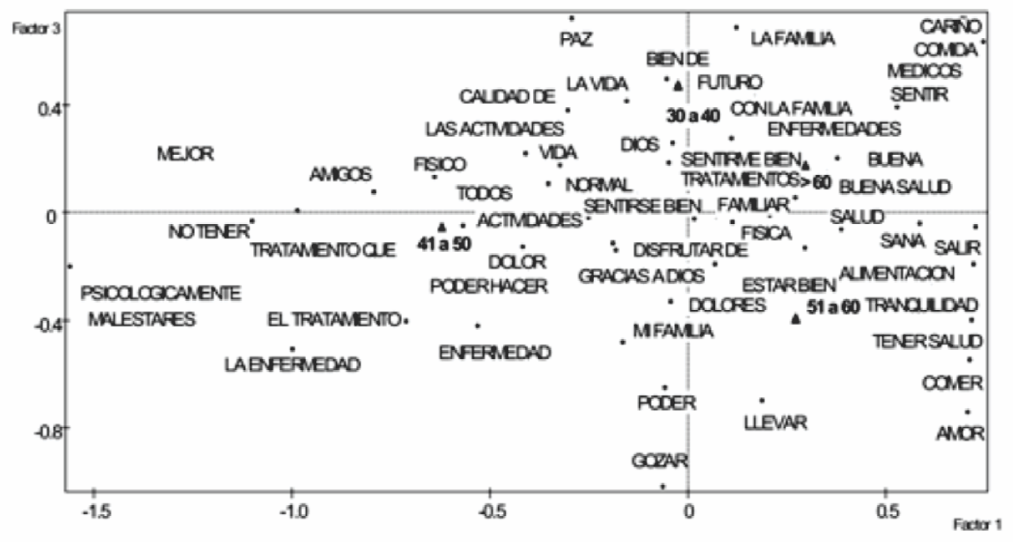


La evaluación de las formas léxicas características apoya la multidimensionalidad del concepto de calidad de vida, ya que se incorporan aspectos clásicamente propuestos como ausencia o minimización de síntomas, bienestar psíquico, autonomía, funcionalidad y bienestar familiar $(11,24)$. Un hallazgo llamativo del presente estudio es la presencia de dos dominios adicionales: el espiritual y el relacionado con acceso a servicios de salud que garanticen un adecuado tratamiento. El dominio espiritual ya ha sido previamente propuesto (38-41); adicionalmente se ha destacado la importancia de este dominio en pacientes de grupos de edad avanzada (40), lo cual coincide con nuestros hallazgos ; sin embargo, el acceso a servicios de salud que garanticen el tratamiento (hallazgo en los grupos de edad más jóvenes) no ha sido un dominio habitualmente descrito en otras investigaciones en esta área.

El análisis de correspondencias binarias sugiere la presencia de tres dimensiones constitutivas del concepto de calidad de vida: i) Salud no solo como ausencia de síntomas y enfermedad, sino como capacidad de disfrutar funciones nutricias y afectivas, ii) Capacidad de autonomía o de sana dependencia, iii) Armonía familiar y social. La primera dimensión tiene algunas coincidencias con la definición estándar de salud de la OMS. La segunda dimensión se relaciona con la capacidad funcional del paciente, elemento considerado en diferentes instrumentos para medir calidad de vida; incluso, en algunos estudios se utilizan escalas de funcionalidad como equivalentes de calidad de vida $(6,14)$. La tercera dimensión se relaciona con el ambiente familiar y social que también es incorporado en varios instrumentos $(42,43)$. Adicionalmente se encontró que en cada una de estas tres dimensiones se proyectan de manera diferencial los grupos de edad: el componente de calidad de vida relacionado con salud, concebida como ausencia de síntomas y como capacidad de disfrute de funciones nutricias y afectivas, es predominante en grupos de edad intermedios; la capacidad funcional parece valorarse más en los grupos de pacientes mayores de 60 años; los aspectos relacionados con armonía familiar y social son más valorados en grupos de pacientes de 30 a 40 años. Esto sugiere que la calidad de vida no es una dimensión estática, sino cambiante, siendo la edad uno de los factores determinantes de su estructura dinámica. Los hallazgos de nuestra investigación ayudarían cuestionar la utilidad de los instrumentos estandarizados para medir la calidad de vida relacionada con la salud.

Como limitaciones del presente estudio anotamos que no se incorporaron otros aspectos que pudieran relacionarse con la subjetividad del constructo, como pueden ser el estadio de la enfermedad, el tipo de cáncer, el sexo, características del tratamiento y la presencia de comorbilidad entre otros. 
Podemos concluir del presente estudio lo siguiente:

- Los conceptos de salud y bienestar son los más frecuentemente relacionados con el constructo de calidad de vida.

- Además de las dimensiones tradicionales en este estudio emergen la espiritualidad y la disponibilidad de sistemas de salud que garanticen el tratamiento como dominios importantes en pacientes con cáncer.

- La calidad de vida es un concepto multidimensional y dinámico que se modifica dependiendo de la edad de los pacientes. Este elemento apoya la concepción subjetiva del constructo.

- Dada la subjetividad y naturaleza dinámica del constructo, puede ser cuestionable la utilidad de las escalas desarrolladas para su medición •

Agradecimientos: A la Facultad de Medicina de la Universidad Nacional de Colombia y al Instituto Nacional de Cancerología por el apoyo ofrecido.

\section{REFERENCIAS}

1. Frost MH, Sloan JA. Quality of life measurements: a soft outcome-or is it? Am J Manag Care. 2002 Dec; 8(18 Suppl):S574-9.

2. Carr AJ, Gibson B, Robinson PG. Measuring quality of life: Is quality of life determined by expectations or experience? BMJ. 2001; May 19. 322 (7 296):1240-3.

3. Macduff $C$. Respondent-generated quality of life measures: useful tools for nursing or more fool's gold? J Adv Nurs. 2000; Aug. 32(2):375-82.

4. Jocham HR, Dassen T, Widdershoven G, Halfens R. Quality of life in palliative care cancer patients: a literature review. J Clin Nurs. 2006; Sep.15(9):1188-95.

5. Jocham HR, Dassen T, Widdershoven G, Halfens RJ. Quality-of-life assessment in a palliative care setting in Germany: an outcome evaluation. Int J Palliat Nurs. 2009; Jul. 15(7):33845.

6. Sánchez R, Ballesteros M, Gómez A. Medición de la calidad de vida en ensayos clínicos de pacientes con cáncer. Un estudio bibliométrico. Rev Col Cancerología. 2009; 13(1):2934.

7. Moons $\mathrm{P}$, Budts W, De Geest S. Critique on the conceptualisation of quality of life: a review and evaluation of different conceptual approaches. Int J Nurs Stud. 2006; Sep. 43(7):891-901.

8. Feinstein AR. Clinimetric perspectives. J Chronic Dis. 1987; 40(6):635-40.

9. Ragsdale D, Kotarba JA, Morrow JR. Quality of life of hospitalized persons with AIDS. Image J Nurs Sch.1992; Winter. 24(4):259-65.

10. Young KM. Where's the evidence? Am J Nurs. 2003; Oct. 103(10):11.

11. Ahmedzai SH, Costa A, Blengini C, Bosch A, Sanz-Ortiz J, Ventafridda V, et al. A new international framework for palliative care. Eur J Cancer. 2004; Oct. 40(15):2192-200.

12. Ferrans CE, Powers MJ. Psychometric assessment of the Quality of Life Index. Res Nurs Health. 1992; Feb. 15(1):29-38.

13. Ferrans CE, Powers MJ. Quality of life index: development and psychometric properties. Rech Soins Infirm. 2007; Mar. (88):32-7.

14. Smith KW, Avis NE, Assmann SF. Distinguishing between quality of life and health status in quality of life research: a meta-analysis. Qual Life Res.1999; Aug. 8(5):447-59. 
15. Albrecht GL, Devlieger PJ. The disability paradox: high quality of life against all odds. Soc Sci Med. 1999; Apr. 48(8):977-88.

16. Skevington SM. Measuring quality of life in Britain: introducing the WHOQOL-100. J Psychosom Res. 1999; Nov. 47(5):449-59.

17. Slevin ML, Plant H, Lynch D, Drinkwater J, Gregory WM. Who should measure quality of life, the doctor or the patient? Br J Cancer. 1988; Jan. 57(1):109-12.

18. Taylor RM, Gibson F, Franck LS. A concept analysis of health-related quality of life in young people with chronic illness. J Clin Nurs. 2008; Jul. 17(14):1823-33.

19. Moons $P$. Why call it health-related quality of life when you mean perceived health status? Eur J Cardiovasc Nurs. 2004; Dec. 3(4):275-7.

20. Meeberg GA. Quality of life: a concept analysis. J Adv Nurs.1993; Jan.18(1):32-8.

21. Bramston P, Chipuer H, Pretty G. Conceptual principles of quality of life: an empirical exploration. J Intellect Disabil Res. 2005; Oct. 49(Pt 10):728-33.

22. Bradley C. Importance of differentiating health status from quality of life. Lancet. 2001; Jan 6;357(9249):7-8

23. Skevington SM. Advancing cross-cultural research on quality of life: observations drawn from the WHOQOL development. World Health Organisation Quality of Life Assessment. Qual Life Res. 2002; Mar.11(2):135-44.

24. Skevington SM, Sartorius N, Amir M. Developing methods for assessing quality of life in different cultural settings. The history of the WHOQOL instruments. Soc Psychiatry Psychiatr Epidemiol. 2004; Jan. 39(1):1-8.

25. Higginson IJ, Carr AJ. Measuring quality of life: Using quality of life measures in the clinical setting. BMJ. 2001; May 26. 322(7297):1297-300.

26. Griffin AM, Butow PN, Coates AS, Childs AM, Ellis PM, Dunn SM, et al. On the receiving end. V: Patient perceptions of the side effects of cancer chemotherapy in 1993. Ann Oncol.1996; Feb. 7(2):189-95.

27. Zhan L. Quality of life: conceptual and measurement issues. J Adv Nurs.1992; Jul. 17(7):795800.

28. Carr AJ, Higginson IJ. Are quality of life measures patient centred? BMJ. 2001; Jun 2. 322(7298):1357-60.

29. Bowling A. What things are important in people's lives? A survey of the public's judgements to inform scales of health related quality of life. Soc Sci Med. 1995; Nov. 41(10):144762.

30. Bernheim JL. How to get serious answers to the serious question: "How have you been?": subjective quality of life (QOL) as an individual experiential emergent construct. Bioethics.1999; Jul. 13(3-4):272-87.

31. Chambers LW, Macdonald LA, Tugwell P, Buchanan WW, Kraag G. The McMaster Health Index Questionnaire as a measure of quality of life for patients with rheumatoid disease. J Rheumatol.1982; Sep-Oct. 9(5):780-4.

32. Dazord A, Leizorovicz A, Gerin P, Boissel JP. Quality of life of patients during treatment of type I diabetes. Importance of a questionnaire focused on the subjective quality of life. Diabete Metab.1994; Sep-Oct. 20(5):465-72.

33. Pineros M, Hernandez G, Bray F. Increasing mortality rates of common malignancies in Colombia: an emerging problem. Cancer2004 Nov 15;101(10):2285-92.

34. Neyt M, Albrecht $\mathrm{J}$. The long-term evolution of quality of life for disease-free breast cancer survivors: a comparative study in Belgium. J Psychosoc Oncol. 2006; 24(3):89-123.

35. Petersen LR, Clark MM, Novotny P, Kung S, Sloan JA, Patten CA, et al. Relationship of optimism-pessimism and health-related quality of life in breast cancer survivors. $\mathrm{J}$ Psychosoc Oncol. 2008; 26(4):15-32.

36. Bécue M. Análisis de datos textuales. Métodos estadísticos y algoritmos. Paris: Cisia; 1991.

37. Lebart L, Salem A, Berry L. Exploring textual data. Dordrecht; Boston: Kluwer Academic; 1998. 
38. Whitford HS, Olver IN, Peterson MJ. Spirituality as a core domain in the assessment of quality of life in oncology. Psychooncology. 2008; Nov. 17(11):1121-8.

39. O'Connell KA, Skevington SM. To measure or not to measure? Reviewing the assessment of spirituality and religion in health-related quality of life. Chronic IIIn. 2007; Mar. 3(1):7787.

40. Molzahn AE. Spirituality in later life: effect on quality of life. J Gerontol Nurs. 2007; Jan. 33(1):32-9.

41. Brady MJ, Peterman AH, Fitchett G, Mo M, Cella D. A case for including spirituality in quality of life measurement in oncology. Psychooncology. 1999; Sep-Oct. 8(5):417-28.

42. Aaronson NK, Ahmedzai S, Bergman B, Bullinger M, Cull A, Duez NJ, et al. The European Organization for Research and Treatment of Cancer QLQ-C30: a quality-of-life instrument for use in international clinical trials in oncology. J Natl Cancer Inst. 1993; Mar 3. 85(5):365-76.

43. Webster K, Cella D, Yost K. The Functional Assessment of Chronic Illness Therapy (FACIT) Measurement System: properties, applications, and interpretation. Health Qual Life Outcomes. 2003; 1:79. 